\title{
REDUCTION OF QUASI-LATTICES TO LATTICES
}

\author{
C. Ganesa Moorthy ${ }^{a}$ and SG. Karpagavalli ${ }^{b}, *$ \\ ${ }^{a}$ Department of Mathematics, Alagappa University, Karaikudi-630 004, \\ Tamil Nadu, India. \\ ${ }^{b}$ Department of Mathematics, Vidhyaa Giri College of Arts and Science, Puduvayal-630 \\ 108, Tamil Nadu, India. \\ E-mail: ganesamoorthyc@gmail.com (C. Ganesa Moorthy) \\ sgkarpa@gmail.com (SG.Karpagavalli)
}

\begin{abstract}
Quasi-lattices are introduced in terms of 'join' and 'meet' operations. It is observed that quasi-lattices become lattices when these operations are associative and when these operations satisfy 'modularity' conditions. A fundamental theorem of homomorphism proved in this article states that a quasi-lattice can be mapped onto a lattice when some conditions are satisfied.
\end{abstract}

Key words: Minimal upper bound, Congruence relation, Partition. AMS Subject Classification(2010): 06B10,18B35,06C05.

\section{Introduction}

The concept of a minimal upper bound is not widely known. A lattice is a partially ordered set (poset) in which any two elements have a least upper bound and a greatest lower bound. A quasi-lattice is a poset in which any two elements have a minimal upper bound and a maximal lower bound. Every quasi-lattice is a lattice. This article tries to establish fundamental facts about quasi-lattices. But, it finds that associativity of 'meet' and

${ }^{*}$ Corresponding author 
'join' operations of quasi-lattices is a unique property of lattices. Similarly it is established that 'modularity' is also a unique property of lattices. A fundamental theorem of homomorphism found in this article also reduces quasi-lattices into lattices. The books [3] and [2] are referred to fundamental definitions and properties for posets and lattices. Although there are many recent articles (see, for example $[4,5,6]$ ) the results of these articles will not be extended to quasi-lattices, because quasi-lattices reduce to lattices when some fundamental properties are assumed.

A partial order $\leq$ on a non empty set $P$ is a relation that is reflexive, anti-symmetric and transitive. A poset $(P, \leq)$ is a non empty set $P$ with a partial order $\leq$. An element $a$ in a partially ordered set $(P, \leq)$ is a maximal lower bound of a non empty subset $A$ of $P$ if $a \leq x, \forall x \in A$, and if there is no element $d$ in $P$ such that $a<d \leq x, \forall x \in A$.Dually a minimal upper bound is defined. A partially ordered set $(P, \leq)$ is called quasi-lattice, if any two elements of $P$ have a minimal upper bound and a maximal lower bound. However, two elements in a quasi-lattice may have more than one maximal lower bound and may have more than one minimal upper bound. Let us use the notations $x \wedge y$ and $x \vee y$ to denote some (particular) maximal lower bound and some minimal upper bound of $x$ and $y$, respectively, in a partially ordered set.

Example 1.1 The Hasse diagram given in the Figure 1 represents a quasilattice. In this diagram the point $x \vee(y \vee z)$ represents another minimal upper bound of $\{x, y\}$ in addition to $x \vee y$. So, it is not a lattice. In this quasi-lattice, $(x \vee y) \vee z$ can never take " the form" $x \vee(y \vee z)$. So, associativity fails to be true.

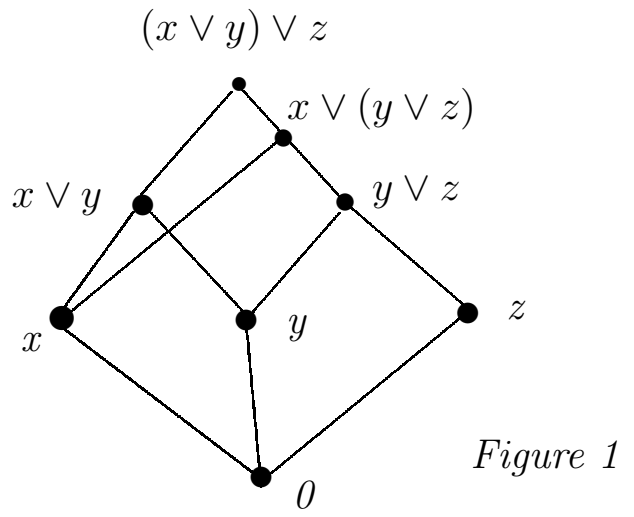




\section{Associative quasi-lattices}

It would be difficult to derive many results for quasi-lattices, when associativity is not assumed.

Definition 2.1 A quasi-lattice $(P, \leq)$ is called an associative lattice, if

(i) $a \vee(b \vee c)=(a \vee b) \vee c$, and

(ii) $a \wedge(b \wedge c)=(a \wedge b) \wedge c$ hold for every $a, b, c \in P$.

Here (i) means that if $a_{1}$ is a minimal upper bound of $\{b, c\}$ and if $a_{2}$ is a minimal upper bound of $\left\{a, a_{1}\right\}$, then there is a minimal upper bound $a_{3}$ of $\{a, b\}$ such that $a_{2}$ is a minimal upper bound of $\left\{a_{3}, c\right\}$ and similarly; if $b_{1}$ is a minimal upper bound of $\{a, b\}$ and $b_{2}$ is minimal upper bound of $\left\{b_{1}, c\right\}$, then there is a minimal upper bound $b_{3}$ of $\{b, c\}$ such that $b_{2}$ is a minimal upper bound of $\left\{a, b_{3}\right\}$. This interpretation clarifies the meanings for the present

notation. When this is followed, the meaning of the following proposition is unambigious.

Proposition 2.2 The following identities are true in a quasi-lattice $(P, \leq)$. (A1): $a \vee a=a ;(A 2): a \wedge a=a ;(A 3): a \vee b=b \vee a ;(A 4): a \wedge b=b \wedge a$; $(A 5): a \vee(a \wedge b)=a=(a \wedge b) \vee a ;(A 6): a \wedge(a \vee b)=a=(a \vee b) \wedge a$; $\forall a, b \in P$.

Proof: Let us verify $a \vee(a \wedge b)=a$. Let $a_{1}$ be a maximal lower bound of $\{a, b\}$, and $a_{2}$ be a minimal upper bound of $\left\{a_{1}, a\right\}$. Then $a_{2}=a$ because $a_{1} \leq a$. Other relations can also be verified in this way.

If $(P, \leq)$ is an associative quasi-lattice, then it further has the properties: $(\mathrm{A} 7): a \vee(b \vee c)=(a \vee b) \vee c$ and $(\mathrm{A} 8): a \wedge(b \wedge c)=(a \wedge b) \wedge c ; \forall a, b, c \in P$. It is known that the relations ( A1) to (A8) characterize a lattice, when $a \vee b$ and $a \wedge b$ are unique elements ( see Theorem 1 in Section 1 in Chapter 1 in [2] ). It is to be proved that an associative quasi-lattice should be a lattice. For this purpose, let us introduce some changes in applications of the notations $\vee$ and $\wedge$. For a given poset $(P, \leq), A \subseteq P$ and $B \subseteq P$, let $A \vee B$ (respectively, $A \wedge B$ ) denote the collection of all elements of the form $a \vee b$ (respectively, $a \wedge b$ ) with $a \in A$ and $b \in B$. So, for example, the relation $a \wedge(a \vee b)=a=(a \vee b) \wedge a$ will mean $\{a\} \wedge(\{a\} \vee\{b\})=\{a\}=(\{a\} \vee\{b\}) \wedge\{a\}$. Thus a poset $(P, \leq)$ is a quasi-lattice if and only if $\{a\} \vee\{b\}$ and $\{a\} \wedge\{b\}$ are non empty subsets of $P$, for any $a, b \in P$. It is a lattice if and only if $\{a\} \vee\{b\}$ and $\{a\} \wedge\{b\}$ are singleton subsets of $P$, for any $a, b \in P$. 
Theorem 2.3 A quasi-lattice $(P, \leq)$ is associative if and only if it is a lattice.

Proof: Suppose $(P, \leq)$ is an associative quasi-lattice. Let $x, y \in P$ and $a, b \in\{x\} \wedge\{y\}$.Then $a \leq y, a \leq x$ and $(\{x\} \wedge\{y\}) \wedge\{a\}=\{x\} \wedge(\{y\} \wedge\{a\})=$ $\{x\} \wedge\{a\}=\{a\}$, when $(\{x\} \wedge\{y\}) \wedge\{a\} \supseteq\{a, b\} \wedge\{a\}=\{a\} \cup(\{b\} \wedge\{a\})$. Thus $\{a\} \wedge\{b\}=\{a\}$ so that $a \leq b$. Similarly $b \leq a$ so that $a=b$. Thus $\{x\} \wedge\{y\}$ contains a unique element. Dually, $\{x\} \vee\{y\}$ contains a unique element. This proves that $(P, \leq)$ is a lattice.

\section{Modular quasi-lattices}

Definition 3.1 A quasi-lattice $(P, \leq)$ is said to be modular if $\{x\} \vee(\{y\} \wedge$ $\{z\})=(\{x\} \vee\{y\}) \wedge\{z\}$ whenever $x, y, z \in P$ and $x \leq z$.

Theorem 3.2 A modular quasi-lattice $(P, \leq)$ is a lattice.

Proof: Fix $x, y$ in the given modular lattice $(P, \leq)$. Let $a, b \in\{x\} \wedge\{y\}$. Then $a \leq x, a \leq y, b \leq x$, and $b \leq y$. So, $(\{x\} \wedge\{y\}) \vee\{a\}=\{a\} \vee(\{y\} \wedge$ $\{x\})=(\{a\} \vee\{y\}) \wedge\{x\}=\{y\} \wedge\{x\}=\{x\} \wedge\{y\}$, when $\{a, b\} \subseteq\{x\} \wedge\{y\}$. So $\{a, b\} \vee\{a\} \subseteq\{x\} \wedge\{y\}$ and hence $\{a, a \vee b\} \subseteq\{x\} \wedge\{y\}$. Thus $a \vee b \in\{x\} \wedge\{y\}$, when $a \vee b \geq a, a \vee b \geq b, a \in\{x\} \wedge\{y\}$ and $b \in\{x\} \wedge\{y\}$. So,the maximality of $a$ and $b$ implies that $a=a \vee b=b$. In particular, $\{x\} \wedge\{y\}$ contains atmost one point. Dually $\{x\} \vee\{y\}$ contains atmost one point. This proves the theorem.

Associative quasi-lattices are lattices and modular quasi-lattices are lattices. So it is difficult to derive new results for quasi-lattices, because quasilattices with additional fundamental properties become lattices. However, one can derive fundamental results for ideals.

Definition 3.3 A subset I ( $\mathscr{F}$, respectively) of a quasi-lattice $(P, \leq)$ is called an ideal (a filter, respectively), if

(i) $a, b \in I \Rightarrow\{a\} \vee\{b\} \subseteq I$

((i) $a, b \in \mathscr{F} \Rightarrow\{a\} \wedge\{b\} \subseteq \mathscr{F}$, respectively) and

(ii) $a \in I, b \in P, b \leq a \Rightarrow b \in I$

((ii) $a \in \mathscr{F}, b \in P, b \geq a \Rightarrow b \in \mathscr{F}$, respectively). 
An arbitrary intersection of ideals (filters) in a quasi-lattice is an ideal (a filter). The intersection of a filter and an ideal is sub quasi-lattice. Here, a sub quasi-lattice $(Q, \leq)$ of a quasi-lattice $(P, \leq)$ means that $\{x\} \vee\{y\} \subseteq Q$, and $\{x\} \wedge\{y\} \subseteq Q$, whenever $x, y \in Q$. The intersection of a filter with an ideal is a convex subset in view of the following (usual) definition.

Definition 3.4 A subset $C$ of a quasi-lattice $(P, \leq)$ is said to be convex, if $a \in C$, whenever $x, y \in C, a \in P$ and $x \leq a \leq y$.

Notation 3.5 To each $A \subseteq P$, a quasi-lattice, let $(A]$ and $[A)$ denote the smallest ideal and the smallest filter, respectively, containing $A$. They exist in view of the previous remark.

Proposition 3.6 Let $(P, \leq)$ be a quasi-lattice. Let $I(P)$ (respectively, $F(P)$ ) be the collection of all ideals (respectively, filters) of $(P, \leq)$. Then $I(P)$ (respectively, $F(P)$ ) is a complete lattice under the inclusion relation (respectively, inverse inclusion relation).

Proof: Let $\left(I_{\lambda}\right)_{\lambda \in A}$ be a collection of ideals in $P$, Then $\cap\left\{I_{\lambda}: \lambda \in A\right\}$ and $\left(\cup\left\{I_{\lambda}: \lambda \in A\right\}\right]$ are ideals which are the greatest lower bound and the least upper bound of the given collection. A similar argument is applicable for filters.

\section{Congruence relations}

Ideals are associated with inverse image of a least element for a lattice homomorphism. A lattice homomorphism is associated with a congruence. Let us first define a congruence relation for a quasi-lattice.

Definition 4.1 Let $(P, \leq)$ be a quasi-order lattice. An equivalence relation $\theta$ on $P$ is denoted by $x \equiv y(\bmod \theta)$ when $x$ and $y$ are related in $P$ by $\theta$. Moreover, for subsets $A, B$ of $P$, the identity $A \equiv B(\bmod \theta)$ means the following:

(i) to each $a \in A$, there is $a b \in B$ such that $a \equiv b(\bmod \theta)$, and

(ii) to each $b \in B$, there is an $a \in B$ such that $a \equiv b(\bmod \theta)$. 
The equivalence relation $\theta$ on $P$ is called a congruence relation, if $\left\{x_{1}\right\} \wedge$ $\left\{y_{1}\right\} \equiv\left\{x_{2}\right\} \wedge\left\{y_{2}\right\}(\bmod \theta)$, and $\left\{x_{1}\right\} \vee\left\{y_{1}\right\} \equiv\left\{x_{2}\right\} \vee\left\{y_{2}\right\}(\bmod \theta)$, whenever $x_{1} \equiv x_{2}(\bmod \theta)$ and $y_{1} \equiv y_{2}(\bmod \theta)$ in $P$, and if $\{x\} \wedge\{y\} \subseteq[z]$, when $z \in\{x\} \wedge\{y\}$ and $\{x\} \vee\{y\} \subseteq[z]$, when $z \in\{x\} \vee\{y\}$, for $x, y$, z in $P$, when $[z]$ refers to the equivalence class containing $z$, determined by $\theta$.

It is known that the collection of all partitions is a complete lattice under the "refinement" relation. The collection of all congruences on a lattice is a (complete) sublattice of the lattice of all partitions. In the same way(see the proof of theorem 3.9 in [1]), one can verify that the collection of all congruences on a quasi-lattice is a complete lattice and a sublattice of the lattice of all partitions.

Lemma 4.2 Let $(P, \leq)$ be a quasi-lattice, and $\theta$ be a congruence relation on $P$. If $u \equiv v(\bmod \theta), a \in\{u\} \wedge\{v\}, b \in\{u\} \vee\{v\}$, and if $a \leq x \leq b$, then $u \equiv x(\bmod \theta)$.

Proof: Under the assumptions, we have $\{x\}=\{x\} \vee\{a\} \equiv\{x\} \vee(\{u\} \wedge$ $\{v\}) \equiv\{x\} \vee(\{u\} \wedge\{u\}) \equiv(\{x\} \vee\{u\})(\bmod \theta)$. Dually, we have $\{x\}=$ $\{x\} \wedge\{b\} \equiv\{x\} \wedge(\{u\} \vee\{v\}) \equiv\{x\} \wedge(\{u\} \vee\{u\}) \equiv\{x\} \wedge\{u\}(\bmod \theta)$. So, we have $\{u\}=\{u\} \wedge(\{u\} \vee\{x\})=\{u\} \wedge\{x\} \equiv\{x\}(\bmod \theta)$. This proves the lemma.

Definition 4.3 Let $T: P_{1} \rightarrow P_{2}$ be a mapping from a quasi-lattice $P_{1}$ into a quasi-lattice $P_{2}$. It is said to be a q-lattice homomorphism, if $T(\{x\} \vee\{y\})=$ $\{T(x)\} \vee\{T(y)\}$ and $T(\{x\} \wedge\{y\})=\{T(x)\} \wedge\{T(y)\}, \forall x, y \in P$

Definition 4.4 Let $\theta$ be an equivalence relation on a quasi-lattice $(P, \leq)$. Let $[x]$ denote the equivalence class containing $x$. Let us say that $\theta$ satisfies the condition $\left(^{*}\right)$ if the following are true in $P$ :

(i) If $[x] \neq[y], x \leq z$ and $y \leq z$, then there are elements $a \in[x]$ and $b \in[y]$ and there is an element $d \in\{a\} \vee\{b\}$ such that $d \leq z$.

(ii) If $[x] \neq[y], x \geq z$ and $y \geq z$, then there are elements $a \in[x]$ and $b \in[y]$ and there is an element $d \in\{a\} \wedge\{b\}$ such that $d \geq z$.

Let us now state a fundamental theorem of homomorphism. 
Theorem 4.5 Let $(P, \leq)$ be a quasi-lattice. Let $\theta$ be a congruence relation on $P$ that satisfies (*) of definition 4.4. Let $P / \theta$ be the collection of all equivalence classes. Let $[x]$ denote the equivalence class containing $x$. Then $P / \theta$ is a lattice in which we have $[x] \wedge[y]=[x \wedge y]$ and $[x] \vee[y]=[x \vee y]$, for any elements $x \wedge y$ and $x \vee y$ in $\{x\} \wedge\{y\}$ and $\{x\} \vee\{y\}$, respectively. Also, the quotient mapping $\pi: P \rightarrow P / \theta$ defined by $\pi(x)=[x], x \in P$, is a surjective q-lattice homomorphism. On the other hand if $T: P \rightarrow L$ is a surjective q-lattice homomorphism from a quasi-lattice $P$ onto a lattice $L$, then $\left\{T^{-1}(a): a \in L\right\}$ defines a partition that leads to a congruence relation satisfying $\left(^{*}\right)$ of definition 4.4 .

\section{Proof:}

First Part: Define $[x] \leq[y]$ if and only if $a \leq b$ for some $a \in[x]$ and some $b \in[y]$. Suppose $a_{1} \in[x]$ and $b_{1} \in[y]$ such that $a_{1} \leq b_{1}$. If $a_{2} \in[x]$, then $a_{1} \equiv a_{2}(\bmod \theta), a_{2} \leq b_{1} \vee a_{2}$ (for any element of this type) and $\left\{b_{1}\right\} \vee\left\{a_{2}\right\} \equiv\left\{b_{1}\right\} \vee\left\{a_{1}\right\} \equiv\left\{b_{1}\right\}(\bmod \theta)$. If $b_{2} \in[y]$, then $b_{1} \equiv b_{2}(\bmod \theta)$, $a_{1} \wedge b_{2} \leq b_{2}$, and $\left\{a_{1}\right\} \wedge\left\{b_{2}\right\} \equiv\left\{a_{1}\right\} \wedge\left\{b_{1}\right\} \equiv\left\{a_{1}\right\}(\bmod \theta)$. Thus, if $[x] \leq[y]$, then for any $a_{1} \in[x]$, there is a $b_{1} \in[y]$ such that $a_{1} \leq b_{1}$ and for any $b_{2} \in[y]$ there is an $a_{2} \in[x]$ such that $a_{2} \leq b_{2}$. Now let us verify that this relation in $P / \theta$ is a partial order relation. Since $x \leq x$, we have $[x] \leq[x], \forall x \in P$. To prove anti-symmetricity, assume that $[x] \leq[y]$ and $[y] \leq[x]$ for two elements $x, y \in P$. Then there is an element $y_{1} \in[y]$ such that $x \leq y_{1}$; and there is an element $x_{1} \in[x]$ such that $y_{1} \leq x_{1}$. Thus $x \leq y_{1} \leq x_{1}$ and $x_{1} \equiv x$ $(\bmod \theta)$. By the previous lemma 4.2 it is concluded that $y_{1} \equiv x(\bmod \theta)$. This proves that $\leq$ is anti-symmetric in $P / \theta$. To prove transitivity, assume that $[x] \leq[y]$ and $[y] \leq[z]$ for some $x, y, z \in P$. Then there is an element $y_{1} \in[y]$ and there is an element $z_{1} \in[z]$ satisfying $x \leq y_{1} \leq z_{1}$ so that $x \leq z_{1}$, So $(P / \theta, \leq)$ is a poset. To prove that $P / \theta$ is a lattice, consider an element $a \in\{x\} \wedge\{y\}$, for some fixed elements $x, y$. Then $a \leq x$ and $a \leq y$. So $[a] \leq[x]$ and $[a] \leq[y]$. Suppose $[b] \leq[x]$ and $[b] \leq[y]$ for some element $b$ of $P$, and assume that $[a] \leq[b]$. Then there is an element $b_{1} \in[b]$ such that $a \leq b_{1}$. There are elements $c_{1} \in[x]$ and $c_{2} \in[y]$ such that $b_{1} \leq c_{1}$ and $b_{1} \leq c_{2}$. By the condition $(*)$ satisfied, there are elements $a_{1} \in\left[c_{1}\right]$ and $a_{2} \in\left[c_{2}\right]$ and there is an element $d \in\left\{a_{1}\right\} \wedge\left\{a_{2}\right\}$ such that $b_{1} \leq d$. Since $\{a\} \equiv\{x\} \wedge\{y\} \equiv\left\{c_{1}\right\} \wedge\left\{c_{2}\right\} \equiv\left\{a_{1}\right\} \wedge\left\{a_{2}\right\} \equiv\{d\}(\bmod \theta)$, we have the relation $[b] \leq[a]$. Thus $[a]=[b]$. This proves that $[x] \wedge[y]=[x \wedge y]$ for any element $x \wedge y, \forall x, y \in P$. Dually, one can prove that $[x] \vee[y]=[x \vee y]$, for any element $x \vee y, \forall x, y \in P$. So, $P / \theta$ is a lattice. Other sub divisions of the 
first part are trivial.

Second Part: Let $\theta$ be the equivalence relation induced by the partition $\left\{T^{-1}(a): a \in L\right\}$. The condition $(*)$ of definition 4.4 has to be checked to complete the proof as the other sub divisions are trivial. If $T^{-1}(a) \neq T^{-1}(b)$, $a_{1} \in T^{-1}(a), b_{1} \in T^{-1}(b), d_{1} \geq a_{1}, d_{1} \geq b_{1}$ and $T\left(d_{1}\right)=d$, then $d \geq a \vee$ $b,\left\{a_{1}\right\} \vee\left\{b_{1}\right\} \subseteq T^{-1}(a \vee b)$, and $\left[a_{1} \vee b_{1}\right] \leq\left[d_{1}\right]$ (in view of the order relation introduced in first part) so that there are $a_{2} \in T^{-1}(a), b_{2} \in T^{-1}(b)$ such that $d_{1} \geq a_{2} \vee b_{2} \equiv a_{1} \vee b_{1}(\bmod \theta)$. Similarly, if $T^{-1}(a) \neq T^{-1}(b), a_{1} \in T^{-1}(a), b_{1} \in$ $T^{-1}(b), d_{1} \leq a_{1}$ and $d_{1} \leq b_{1}$ then there are $a_{2} \in T^{-1}(a), b_{2} \in T^{-1}(b)$, such that $d_{1} \leq a_{2} \wedge b_{2} \equiv a_{1} \wedge b_{1}(\bmod \theta)$. This completes the proof of the theorem.

\section{References}

[1] T.S.Blyth, Lattices and ordered structures, Springer, London, 2005.

[2] G.Gratzer, General lattice theory, Academic press, New York, 1978.

[3] E.Harzheim, Ordered sets, Springer, New York, 2005.

[4] E.T.Schmidt, Semimodular lattices and the Hall-Dilworth gluing construction, Acta.Math.Hungar, 127(3)(2010) 220-224.

[5] P.Terraf, Factor congruences in semilattices, Revista de la Union Mathematica Argentina, 52(2011) 1-10.

[6] F.Wehrung , A solution to Dilworth's congruence lattice problem, Advances in Mathematics, 216(2007) 610-625. 